\title{
Live surgical demonstration: is it worthwhile?
}

\author{
Bertrand Guillonneau
}

An invited professorship is a prestigious opportunity in an academic career. Visiting professors educate through face-to-face communication and, for surgeons, through surgical demonstrations. An operative demonstration allows the visiting surgeon to present the protocol of an operation to a surgical team, the practical use of specific instruments, and the execution of particular techniques. Observing live procedures helps everyone to better appreciate the subtleties of technique and skill. A surgeon's selfexposure means taking risks, but by revealing encountered difficulties it is also possible to demonstrate how to manage those difficulties, thus showing expertise. Nevertheless, safeguards are needed to prevent live surgery slipping from medical education to medical spectacle. The proliferation of surgery video viewing in many conferences, grand rounds, meetings, debates, and on the internet has revitalized the practice of live surgery observation, as attendance is no longer restricted to a small team. These procedures have become indispensable to some national and international meetings. A once rare event for a privileged few, the live operation has arguably become trivialized by overexposure.

Performing surgery in an unfamiliar operating room brings numerous challenges and might put patients at risk. When complications do arise, it is reasonable to question the quality of care given under 'guest' conditions, and legal issues should be considered. Whatever they might think, the invited professor is the surgeon responsible, even if they are not listed as the primary surgeon and are not named on the informed consent documents. The invited surgeon has agreed to take the patient into their care, perform the operation, and therefore implicitly to assume responsibility. Active surgical care of the patient continues after the invited professor returns to their own department, and it is important for the surgeon

\section{Performing \\ surgery in an \\ unfamiliar \\ operating \\ room brings \\ numerous \\ challenges \\ and might put \\ patients at risk.}

B Guillonneau is

Head of the Section of

Minimally Invasive

Surgery, within

the Department of

Urology at Memorial

Sloan-Kettering

Cancer Center,

New York, NY, USA.

Competing interests

The author declared he has no competing interests.

www.nature.com/clinicalpractice doi:10.1038/ncpuro0728 to remain involved with the patient's care following surgery. In cases where complications arise, forgetting this responsibility could lead the invited professor and the hospital into complicated legal situations.

As patients' expectations of positive results grow, performing an operation at a distant or foreign institution for education purposes seems increasingly archaic and risky, and I wonder whether we should explore other options that might ensure a good level of surgical education whilst limiting the risks for patients and surgeons involved.

Video transmission of a surgical procedure from the surgeon's institution to a conference room anywhere in the world is the simplest and most efficient way for live teaching of surgery. Spectators can see what the surgeon sees, and, by working in their own environment, the surgeon can ensure that the patient receives the best possible care. One problem with this way of demonstrating surgical procedures is that often only the operative part of the surgical procedure is recorded, but this can be easily remedied. Video recording of surgery that is then presented to the audience by the surgeon is another way of surgical teaching. The immediacy of the live operation is lost, but this has the advantage of being more interactive, since the video can be replayed at any time to review a technique, an anatomic detail, or a surgical situation. What we lose in spectacle, we gain in education.

It is essential to raise the question of the riskto-benefit ratio of performing live surgery in an unfamiliar environment. There is little doubt that the morbidity is higher, and that it is difficult to ensure the continuity of care. We must continue to share our knowledge of the technical methods available, and it appears unreasonable in our world of advanced technology to continue to put patients at risk of complications and surgeons at risk of legal challenges. 